Department of Health Services for the Elderly, Ministry of Health and Welfare, for promotion of this study.

1 Department of Health Services for the Elderly, Ministry of Health and Welfare. Rojin hoken jigyo no susumekata: toshibu ni okeru torikumi. Tokyo: Gyosei, 1990.

2 Breslow L. Prospects for improving health through reducing risk factors. Prev Med 1978;7:449-58.

3 Kosei Tokei Kyokai. Kokumin eisei no doko, 1990. Tokyo: Kosei Tokei Kyokai, 1990.
4 Secretaries of State for Social Services, Wales, Northern Ireland, and Scotland. Promoting better health. The government's programme for improving priman Promoting better health. The gover
health care. London: HMSO, 1987.

5 Roworth MA. Screening in the elderly. Public Health 1989;103:377-83

6 Tatara K, Shinsho F, Asakura S, Hashimoto M, eds. Shi-cho-son no hoken jigvo. Tokyo: Nihon Koshueisei Kyokai, 1984

7 Marmot MG, Smith GD. Why are the Japanese living longer? BMf 1989;299: $1547-51$.

(Accepted 21 fanuary 1991)
Combined Breast Clinic, St George's Hospital, London SW17 0QT A Yelland, $\mathrm{MB}$, research registrar

M D Graham, FRCs, research registrar

P A Trott, FRCPATH, consultant pathologist

H T Ford, FRCR, consultant radiotherapist

R C Coombes, FRCP, consultant oncologist

J-C Gazet, FRCS, consultant surgeon

N G Polson, PHD, statistician

Correspondence to:

Mr Gazet.

BMf 1991;302:618-20

\title{
Diagnosing breast carcinoma in young women
}

\author{
A Yelland, M D Graham, P A Trott, H T Ford, R C Coombes, J-C Gazet, N G Polson
}

\begin{abstract}
Objective-To assess the individual and combined diagnostic accuracy of clinical examination, mammography, and fine needle aspiration biopsy in young women with breast cancer.

Design-Analysis based on case notes of patients presenting with breast cancer during 1971-89.

Setting-A combined breast clinic.

Patients-Consecutive series of 81 women aged $<36$ with histologically proved breast cancer presenting with a discrete mass over 19 years.

Main outcome measures-Results of clinical examination, xeromammography or conventional mammography, fine needle aspiration biopsy, and examination of tissue removed by surgery.

Results - The clinical diagnosis was correct in $\mathbf{4 7}$ women and radiography in 35 . Fine needle aspiration biopsy was correct in 47 of the 63 women in whom it was successfully performed. Fine needle aspiration was significantly more accurate than mammography $(78 \% v 45 \%, \mathrm{p}<0.01)$. Ten $(16 \%)$ patients had negative results on clinical examination, mammography, and fine needle aspiration.

Conclusion-Mammography alone seems inadequately sensitive to detect breast cancer in young patients. When all investigations give negative results excision biopsy is the only way of obtaining a definitive diagnosis.
\end{abstract}

\section{Introduction}

Mortality from breast cancer has not changed over the past 30 years. Accurate early diagnosis is needed to improve quality of life and reduce mortality as prognosis depends on tumour size at presentation. This is the basis of the national screening programme.

A particularly emotive problem is the diagnosis of carcinoma of the breast in young women. About two thirds of patients presenting to breast clinics are younger than 36; most have benign disease, but roughly $3 \%$ of all carcinomas occur in this age group. Clinical examination, mammography, and fine needle aspiration biopsy are thought to be necessary for diagnosis.' To determine whether this is true we reviewed the methods used to diagnose cancer in patients presenting to our clinic over 19 years.

\section{Patients and methods}

We reviewed a consecutive series of 2820 women with known breast carcinoma presenting between 1971 and 1989 . We found 81 women aged less than 36 with carcinoma proved by histopathological evaluation of a biopsy specimen. Specimens were embedded in paraffin wax and multiple sections stained with haematoxylin and eosin. A consultant histopathologist examined the sections.
All the women presented with a discrete lump in the breast. They were examined by a consultant in our clinic and the clinical diagnosis was recorded as benign or malignant. The general practitioner's diagnosis was also noted. Seventy seven women had mammography. The 62 women seen before October 1986 had xeromammography and the other 15 had standard mammography with a Senograph 500T (IGE, Slough, United Kingdom) machine.

Biopsy specimens were obtained by fine needle aspiration in 67 patients. The technique was always performed by a consultant and in a standard manner. The slides were examined by a consultant pathologist. The service became available in 1975; 11 patients presented before this, and three presented subsequently but did not have a fine needle aspiration biopsy.

Other details noted were: family history of breast disease, either benign or malignant; use of the contraceptive pill for longer than six months; oestrogen receptor status, a value less than $15 \mathrm{fmol} / \mathrm{mg}$ of cytosol being considered a negative result; histological type of carcinoma; and clinical size of the tumour at presentation.

The data obtained were subjected to a standard binomial test by an independent statistician.

Eighty one randomly selected women who were matched for age and had had benign breast disease (fibroadenoma, fibrocystic disease, duct ectasia, and normal tissue) during the same period where used as controls.

\section{Results}

Of the women aged less than 36 with malignant disease, 61 were aged $31-35$ (table I), and the median age at presentation was 33 . Nine women had a first degree relative who had had malignant breast disease and eight had a relative who had had benign disease. Among the control group, a relative had had malignant disease in eight and benign disease in 13 .

Fifty three women in both groups had taken the contraceptive pill for longer than six months. The oestrogen receptor status was negative in 32 out of the 45 women in which it had been tested since the assay's introduction in 1982

In the women with malignant disease clinicians diagnosed 34 tumours as benign and 47 as malignant. The general practitioners diagnosed 18 as malignant and 53 as benign; the diagnosis was not stated in 10 cases.

\section{CLINICALLY BENIGN}

Fine needle aspiration was performed in 27 patients (two did not have the procedure and five presented before the service was available). Malignancy was identified in $15(56 \%)$. In one woman insufficient tissue was obtained and in another the diagnosis was 
TABLE I-Age, TNM stage, and pathological diagnosis in 81 women aged $<36$ with breast cancer

\begin{tabular}{lr}
\hline & $\begin{array}{c}\text { No of } \\
\text { cases }\end{array}$ \\
\hline Age (years): & \\
$21-25$ & 4 \\
$26-30$ & 16 \\
$31-35$ & 61 \\
TNM stage: & \\
T1 & 20 \\
T2 & 54 \\
T3 & 6 \\
T4 & 1 \\
Pathological diagnosis: & \\
Invasive ductal carcinoma & 69 \\
Ductal carcinoma in situ & 6 \\
Invasive lobular carcinoma & 3 \\
Lobular carcinoma in situ & 2 \\
Medullary carcinoma & 1 \\
\hline
\end{tabular}

suspicious. Thirty three women had radiography (29 xeroradiography, four conventional mammography) and malignancy was diagnosed in eight (24\%). Seven cases were identified by xeroradiography $(24 \%)$ and one by conventional mammography $(25 \%)$. No woman had a positive result on mammography but a negative result on fine needle aspiration biopsy.

\section{CLINICALLY MALIGNANT}

Fine needle aspiration was performed in 40 women, and malignancy was confirmed in $32(80 \%)$. There were four false negative results. In three women insufficient tissue was obtained, and in one the diagnosis was suspicious. One woman did not have fine needle aspiration, and in six the service was not available.

Radiography was performed in 44 women and gave positive results in $27(61 \%)$. Xeromammography was used in 33 cases and conventional mammography in 11. Xeromammography was correct in $18(55 \%)$ cases and conventional mammography in nine $(82 \%)$ cases. Mammography gave positive results in only two women in whom fine needle aspiration gave negative results.

The average clinical tumour size at presentation was $3.0 \mathrm{~cm}$. Invasive ductal carcinoma was the most common pathological diagnosis, followed by ductal carcinoma in situ (table I).

\section{Discussion}

In young women prompt diagnosis and treatment of breast cancer are essential, but it is in this group that diagnosis and treatment are most likely to be delayed because of the large numbers with benign disease. ${ }^{3}$

Clinical examination, radiography, and fine needle aspiration have been proved to be beneficial in patients with breast cancer. ${ }^{+5}$ The women studied, however, have been mainly aged over 36 . In such women clinical examination, radiography, and fine needle aspiration in combination may be up to $99 \%$ accurate in preoperative diagnosis. ${ }^{4}$ Clinical examination and radiography are said to be less accurate in younger women. ${ }^{16}$

We found that a family history of benign or malignant breast disease did not predict the diagnosis. This may be because we studied a young population and first degree relatives had not yet developed the disease. We found no excess among women taking the contraceptive pill. This contrasts with the findings published by the United Kingdom study group.'

Clinical examination diagnosed $47(58 \%)$ tumours as malignant in women aged less than 36 , which compares with $2394(85 \%)$ in all women with breast cancer. These figures are comparable with those found in other studies. ${ }^{6}$ Mammography showed an overall sensitivity of $45 \%$ in younger women compared with a figure of up to $94 \%$ for older patients. ${ }^{8}$ Conventional mammography was more accurate than xeromammography, although this did not reach significance $(95 \%$ confidence interval -0.01 to $0.53, p=0.06$ ), the women examined by the two techniques being otherwise similar. Mammography is gaining in accuracy owing to faster film speeds, improved equipment, and better reporting by radiologists specialised in breast disease. Combined ultrasonography and radiography has been shown to increase diagnostic accuracy. ${ }^{89}$ Its wider application in the under 36 age group, who are known to have dense breasts that decrease mammographic accuracy, ${ }^{10}$ will also improve detection rates.

Ultrasonography of the breast has been performed at this hospital since 1988, though it has been peformed in large numbers of women in only the past six months. We have therefore not included our experience in this study. The current policy of our unit is to perform ultrasonography and radiography on all young patients with a discrete lump and to obtain compression paddle radiographs in those with equivocal findings.

The results of fine needle aspiration were correct in 47 cases, and no false positive result was recorded. If cases reported as suspicious were taken as a positive result (as this should lead to operative biopsy) and cases in which insufficient tissue was obtained were eliminated (as they should lead to a repeat specimen being taken) then the sensitivity rose to $78 \%$. The results in patients undergoing both mammography and fine needle aspiration were directly compared to eliminate bias. Fine needle aspiration proved significantly more accurate than mammography in patients with clinically benign and malignant tumours $(56 \% v$ $24 \%, 95 \%$ confidence interval 0.09 to $0.59 ; \mathrm{p}<0.05$ (benign) and $80 \% v 61 \%, 0.11$ to $0.48 ; \mathrm{p}<0.01$ (malignant)). Table II shows the cross tabulation. Mammography diagnosed carcinoma in only two women in whom fine needle aspiration gave negative results; both women had clinically malignant tumours.

TABLE II-Cross tabulation of results of fine needle aspiration and mammography according to clinical diagnosis ${ }^{\star}$

\begin{tabular}{lcc}
\hline & \multicolumn{2}{c}{ Results of fine needle aspiration } \\
\cline { 2 - 3 } Clinical diagnosis & Positive & Negative \\
\hline Benign: & & \\
$\quad$ Positive on mammography & 9 & 0 \\
$\quad$ Negative on mammography & & 10 \\
Malignant: & 20 & 2 \\
$\quad$ Positive on mammography & 13 & 2 \\
Negative on mammography &
\end{tabular}

*Women for whom insufficient material was obtained by fine needle aspiration are excluded.

tIncludes women in whom diagnosis was suspicious.

When all three methods of diagnosis were used together the sensitivity was $84 \%(53 / 63) ; 10(16 \%)$ patients had negative results in all three tests. Three of these patients presented within two years of the fine needle aspiration biopsy service becoming available, and the seven others presented at irregular intervals throughout the remaining period. All 10 patients had their breast lumps removed because of clinical doubt cast on the diagnosis at follow up.

Other centres have proposed that some patients with a discrete, solitary, and apparently benign lesion could

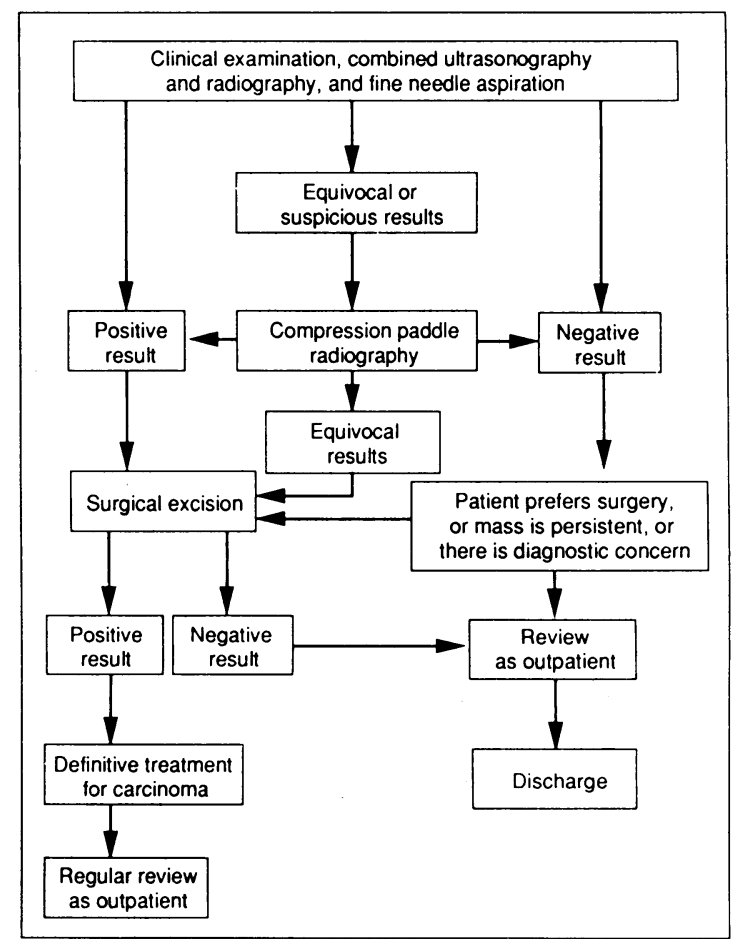

Management of a discrete breast mass in a young patient 
be managed conservatively. ${ }^{31}$ Our results suggest that not only will $16 \%$ of carcinomas be misdiagnosed but a huge burden of outpatient consultations will result if the three monthly follow up policy is adhered to. This conservative policy is also unacceptable to most patients. "

The previous policy of our unit to excise all discrete lumps occurring in the under 36 age group, whatever the preoperative diagnosis, would seem to be supported by our figures. A total of 9768 patients aged under 36 with benign breast disease were seen over the 19 years. If each of these patients had had a discrete lump and had had surgical excision the operative burden would have been 10 cases a week. Tru-Cut biopsy could be used to provide tissue provided that representative samples are obtained. Most patients, however, prefer surgical excision."

Our current policy is to perform fine needle aspiration in all women with clinically discrete breast masses. Combined ultrasonography and radiography is also performed in all patients with a discrete mass. If the results of combined mammography are equivocal compression paddle radiographs are taken. As soon as the results are available the patient is reviewed in an assessment clinic. Any concern over the nature of the mass results in excision biopsy being performed (figure).
We suggest that centres not possessing adequate cytological and combined mammographic facilities should excise all discrete breast masses in this age group without previous investigation. We also suggest that the poor detection rate by general practitioners warrants all young patients presenting with a breast lump being referred to a surgeon with an interest in breast disease.

1 Dixon JM, Anderson TJ, Lamb J. Fine needle aspiration cytology in relationship to clinical examination and mammography in the diagnosis of a solid breast mass. Br F Surg 1984;71:593-6.

2 Trott PA, Thomas MJ, Coombes RC, et al. The diagnosis of primary breast cancer in breast cancer management. London: Academic Press, 1981:1-16.

3 Wilkinson S, Forrest APM. Fibro-adenoma of the breast. Br $\mathrm{f}$ Surg $1985 ; 72: 838-40$

4 Thomas MJ, Fitzharris BM, Redding WH, et al. Clinical examination, xeromammography, and fine needle aspiration cytology in the diagnosis of

5 Smallwood J, Herbert A, Guyer PB, Taylor I. Accuracy of aspiration cytology in the diagnosis of breast disease. Brf S Surg 1985;72:841-3.

6 Ashley S, Royle GT, Corder A, et al. Clinical, radiological and cytological diagnosis of breast cancer in young women. Br f Surg 1989;76:835-7.

7 United Kingdom National Case Control Study Group. Oral contraceptive use and breast cancer risk in young women. Lancet 1989;i:973-82.

8 Teixidor HS, Kayan E. Combined mammographic-sonographic evaluation of breast masses. AfR 1977;128:409-17.

9 Warwick DJ, Smallwood JA, Guyer PB, et al. Ultrasound mammography in the management of breast cancer. Br F Surg 1988;75:243-5.

10 Cahill CJ, Boulter PS, Gibbs NM, et al. Features of mammographically negative breast cancer. Brf Surg 1981;68:882-4.

11 Cant PJ, Madden MV, Close PM, et al. Case for conservative management of selected fibroadenomas of the breast. Br f Surg 1987;74:857-9.

(Accepted 10 January 1991)

\title{
Idiopathic first seizure in adult life: who should be treated?
}

\author{
Cees A van Donselaar, Ada T Geerts, Robert-Jan Schimsheimer
}

\section{Abstract}

Objective-To assess the accuracy of the diagnosis, recurrence rate, and fate after the first recurrence in adult patients with an untreated idiopathic first seizure.

Design-Hospital based follow up study.

Setting-One university hospital and three general hospitals in The Netherlands.

Patients - 165 patients aged 15 years or more with a clinically presumed idiopathic seizure; diagnosis was based on a description of the episode according to prespecified diagnostic criteria.

Main outcome measures-Results of additional investigations and follow up regarding the accuracy of the diagnosis; first recurrence; and response to treatment after the first recurrence.

Results-Computed tomography showed major abnormalities in $5.5 \%$ of the patients and follow up led to doubts about the initial clinical diagnosis in another $6 \%$. Cumulative risk of recurrence was $40 \%$ at two years. The cumulative risk of recurrence at two years was $81 \%$ (95\% confidence interval $66 \%$ to $97 \%$ ) in patients with epileptic discharges on a standard or partial sleep deprivation electroencephalogram, $39 \%(27 \%$ to $51 \%)$ in patients with other electroencephalographic abnormalities, and $12 \%(3 \%$ to $21 \%$ ) in patients with normal electroencephalograms. Treatment was initiated in most patients who had one or more recurrences; $40(70 \%)$ patients were completely controlled, eight $(14 \%)$ had sporadic seizures, and nine $(16 \%)$ did not become free of seizures within one year despite treatment

Conclusions-The decision to initiate or delay treatment should be based on electroencephalographic findings.

\section{Introduction}

Opinions differ on the treatment of patients with an idiopathic first seizure. ${ }^{1-4}$ Treating all patients immediately reduces the number of recurrences 5 and might reduce the number of patients with intractable disease in the long term. ${ }^{67}$ This policy might mean, however, that many patients would be treated unnecessarily.

The data required to solve this dilemma are either lacking or a matter of dispute. The reliability and accuracy of the diagnosis of a first seizure are not known,${ }^{8}$ and investigations of the recurrence rate have produced widely diverging results. ${ }^{9-15}$ The fate of patients after the first recurrence has not been investigated. We conducted a hospital based investigation of 165 patients aged 15 years or more with an idiopathic untreated first seizure.

\section{Patients and methods}

We prospectively studied all patients aged 15 years or more with a presumed idiopathic first seizure who were referred to one university hospital and three general hospitals during March 1986 to March 1988 Patients who had had a seizure other than febrile convulsions in the past were excluded, as were patients presenting with a status epilepticus or with a seizure that had lasted more than 30 minutes. We admitted only patients in whom there was no obvious clinical cause for the seizure. ${ }^{16}$ Patients with seizures that may have been induced by sleep deprivation or stress were included, except for those who had experienced extreme conditions such as not sleeping for several days.

The diagnosis was based on the description of the episode according to prespecified diagnostic criteria, ${ }^{8}$ the medical history, and the neurological examination. We previously showed that the reliability of these criteria was good $(x=0.73){ }^{8}$ All patients were discussed by three neurologists before admission.

The four centres referred 226 patients, and 61 were excluded: four because they did not attend for additional investigations and 57 because another
Correspondence to:

BMF 1991;302:620-3 